\title{
Standpunkt
}

\section{Juristische Fachdidaktik 2.0.14: Reichweitendefizitkompensationsstrategien(?)}

\author{
Roland Schimmel"
}

Der ursprüngliche Titel lautete „Reichweitenprobleme“, war mir dann aber nicht optimistisch genug.

Wählt man - ohne Präjudiz für die künftige Geschichtsschreibung - das Jahr 2009 als Bezugspunkt, weil in Jura 2009, S. 579 ff. der wohl erste Aufsatz seit Jahrzehnten erschienen ist, der die juristische Didaktik zentral thematisiert, kann man nach fünf Jahren eine beeindruckende Entwicklung zwischenbilanzieren: Gründung von Instituten und Kompetenzzentren an etlichen Universitäten, Berufung von (zumeist: Junior-)Professoren ${ }^{1}$ auf einschlägig (teil-)ausgewiesene Stellen, Gründung einer Schriftenreihe zum Thema, Erscheinen eines Jahrbuchs und einer Vierteljahresschrift (liegt dem Leser vor), Veranstaltung von Fachtagungen in beinahe monatlicher Folge. Lehrpreise werden ausgelobt und verliehen, Laudationes gehalten, Förderanträge ausgefüllt und begründet usw. - womit nur ein paar recht gut sichtbare Entwicklungen benannt wären. Wichtiger ist gewiss die lokal stattfindende Etablierung neuer Lehr- und Lernformen oder wenigstens das Experimentieren mit solchen. Am wichtigsten ist vermutlich, dass eine Reihe von Lehrenden die nötige Neugier aufgebracht hat, sich auf Neues einzulassen. Die dafür nötige Beweglichkeit in Hirn und Herz ist im veränderungsresistenten juristischen akademischen Betrieb kaum hoch genug zu schätzen.

Dessen ungeachtet These 1: Die Juristische Fachdidaktik hat ein Reichweitenproblem.

Sie erreicht nämlich nicht oder nur mit Mühe ihre zwei vermutlich wichtigsten Adressatengruppen, Professoren und Studenten.

These 1.1: Die juristische Fachdidaktik erreicht fast nicht: die Lehrstuhlinhaber.

Ein Beispiel: Auf der Teilnehmerliste einer aktuellen juristisch-didaktischen Tagung stehen 61 Personen (nicht alle sind anwesend), davon 11 Professoren. Zieht man die Referenten ab, bleiben sieben. Zieht man - unzulässigerweise - die FH-Professoren ab, bleiben fünf. Zieht man - erneut unzulässigerweise - die Besucher aus dem deutschsprachigen Ausland ab, bleiben drei. Nach Abzug der rechtsdidaktisch Vorbefassten sowie der Inhaber einschlägig ausgewiesener Stellen bleibt: keiner. Nach

* Prof. Dr. Roland Schimmel lehrt Wirtschaftsprivatrecht an der Fachhochschule Frankfurt am Main.

1 Diskriminierung von Frauen finde ich widerlich. Unleserliche Texte sind eine Beleidigung des Lesers. BinnenMajuskeln und dergleichen scheinen mir eine Beleidigung intelligenter Leserinnen zu sein. Ich bitte um Verständnis, wenn ich darauf verzichte. Alle TransGenderPersonen sind im Folgenden mitgemeint. Alle abweichenden Standpunkte respektiere ich, ohne sie zu teilen. 
Abzug der Nichtjuristen bleibt: erst recht keiner. Aktive rechtswissenschaftliche Universitätsprofessoren aus Deutschland: keine. Das ist nicht eben viel. Auf eine biologische Lösung dieses Problems zu setzen, erfordert einen langen Atem. Ob diejenigen unter den heutigen Mittelbauern, die den langen Marsch überlebt haben werden, eines Tages innovative Lehrformen praktizieren, ausprobieren, optimieren, wertschätzen und weiterempfehlen, wird man in zehn Jahren bilanzieren können. Vorausgesetzt, die juristische Fachdidaktik hat sich bis dahin nicht als Strohfeuer entpuppt oder die Zahl der Interessierten ist nicht geschrumpft auf die der Gefährten im Herrn der Ringe (wobei offenbleiben kann, ob Zwerge und Halblinge voll zählen oder halb).

These 1.2: Die juristische Fachdidaktik erreicht die Studenten eher mittelbar.

Obwohl Studenten definitionsgemäß die Hauptnutznießer aller Mühen um gute Lehre sind, sind sie am inhaltlichen Austausch bislang eher am Rande beteiligt. Trotz weitgestreuter Einladung sind sie bei den genannten Tagungsveranstaltungen hauptsächlich an der Rezeption, der Kaffeetheke und der Garderobe vertreten, in Gestalt der Verbandsvertreter meist auch mit einem Teilnehmer bei der obligatorischen Diskussionsrunde - aber noch recht wenig als Zuhörer, die selbst in die Debatte eingreifen. Schade. Man könnte sich schließlich auch leicht eine Fachdidaktik vorstellen, die ihre Hauptinteressenten mit ins Boot holt.

Wenn es ein Reichweitenproblem gibt, drängt sich die Frage nach den Ursachen auf. Ich wage zwei oder drei Vermutungen.

Vermutung 1: Für die meisten potenziellen Interessenten fehlt es an Material „zum Anfassen“.

Es gibt noch kein Lehrbuch Didaktik für-ahnungslose - Juristen. Selbst wenn darin mehr von Didaktik die Rede wäre als von juristischer Didaktik - was übrigens nicht sicher ist, aber gut möglich - käme es doch den Lehrenden jetzt und hier entgegen, die sich nicht mit als zu hoch empfundenem Aufwand in die Terminologie einer fremden Wissenschaft einarbeiten müssten. Nun müsste dieses Buch jemand schreiben und verantworten; das kann man herbeiwünschen, aber nicht zwingen. Gelegentlich gibt es indessen schon Material (etwa Jörn Griebel/Levent Sabanogullari, Moot Courts, Baden-Baden 2011 sowie Tanja Henking/Andreas Maurer, Mock Trials, Baden-Baden 2013), bezeichnenderweise aber doch eher zu Einzelaspekten. Es gibt aber noch nicht einmal eine im Netz leicht zugängliche Ressourcensammlung, und sei es auch nur in einem Wiki, in dem die Arbeit der Bestandspflege auf die Schultern der Nutzer verteilt werden könnte. Vielleicht findet das niemand wichtig, vielleicht fühlt sich niemand zuständig; aber auf Dauer wird so die Chance verspielt, halbwegs niedrigschwellig mehr Interessenten zu gewinnen.

Bemerkenswerterweise bringt „die Rechtsdidaktik“ aber nicht nur kaum gut handhabbare Texte für die Lehrenden hervor, sondern auch kaum innovative Text für die Lernenden (Irrtümer vorbehalten). Während das Angebot von Lehrbüchern zu den 
Pflichtfachgebieten über Jahre und Jahrzehnte immer größer und teils kaum noch überschaubar geworden ist, finden sich praktische keine Texte, die einmal einen konzeptionell neuen Weg beschritten. Und welchen Verbreitungsgrad hat etwa das auf Visualisierung setzende Buch von Bernhard Bergmans, Schuldrecht, Berlin 2009, erreicht? Liegt das daran, dass der Schwerpunkt didaktischer Fragen eher bei der Bewältigung der großen Vorlesung liegt als bei der Gestaltung wirklich hilfreichen Lernmaterials, das auch andere Zugänge erlaubte als das klassische große Lehrbuch oder dessen nicht mehr ganz kleiner Bruder, das Lernbuch, die beide im Wesentlichen als Bleiwüste daherkommen?

Vermutung 2: Die institutionellen und wissenschaftlichen Grundlagen „der Rechtsdidaktik" sind derzeit noch schmal.

Während das Feld, das die juristische Fachdidaktik zu beackern haben wird, teils noch vermessen wird, zeichnet sich inhaltlich eine gewisse Zerfaserung ab. Ein Teil der Interessenten will in erster Linie den Anschluss an den Stand der didaktischen Forschung außerhalb der Rechtswissenschaft zu erreichen versuchen, ein anderer Teil verhandelt eher alte und neue Fragen der Juristenausbildungsreformdebatte unter dem willkommenen neuen Etikett der Rechtsdidaktik.

Wählt man zwei Tagungsthemen aus dem März 2014 als Beispiel, wird deutlich, dass mittlerweile schon einigermaßen spezielle Interessen bedient werden: Drei Tage nach Die Promotion in der Rechtswissenschaft in Hamburg kann man in Köln diskutieren über Praktikumsausbildung im Jurastudium - Wie zeitgemäß ist das JAG? Spätestens mit Die Habilitation in der Rechtswissenschaft unter besonderer Berücksichtigung der fachdidaktischen Qualifikation und Betreuung Späthabilitierender (noch nicht angekündigt, aber denkbar, bislang nur Arbeitstitel) wird ein Spezialisierungsgrad erreicht sein, der die Frage aufwirft, ob so die am „Kerngebiet“ juristischer Fachdidaktik interessierten „Einsteiger“ noch angesprochen werden.

Der wissenschaftliche Unterbau der neuen Disziplin ist in seiner Breite noch nicht leicht wahrnehmbar. Die Schriften zur rechtswissenschaftlichen Didaktik versammeln unter der Betreuung eines Dutzends hochkarätiger Herausgeber seit drei Jahren praktisch nur die Tagungsbände einer Tagungsreihe. Juristische Dissertationen mit Schwerpunkt in der Fachdidaktik sucht man dort (und auch an anderer Stelle) vergebens. Was indes auch daran liegt, dass es solche Dissertationen - bislang - kaum gibt. Erscheint einmal eine, kann sie auch nach Jahren noch mit einer Doppelrezension rechnen. Ausnehmend attraktiv scheint der Themenkreis für Doktoranden aber nicht zu sein: Mindestens droht hier interdisziplinäres Arbeiten, eventuell auch Empirie. Beides geht alles andere als schnell. Und ob die scientific community derlei Themen als Eintrittskarte für eine Wissenschaftskarriere zu akzeptieren bereit ist, wird sich erst noch zeigen müssen.

All das ist vermutlich weniger besorgniserregend als vielmehr ganz normal. Allerdings gibt es Anlass anzunehmen, dass die Zeiten für die Rezeption didaktischer Erkenntnisse mal günstiger stehen und mal weniger günstig. Das führt zu der Frage, 
was geschehen wird, wenn der Zauber des Anfangs verflogen ist und die Projektmittel nicht mehr im gleichen Umfang abrufbar sind wie im Augenblick.

Wollte man aus diesen Überlegungen These 2 ableiten: Wir müssen auf unsere Adressaten achten, unterstellte dies kontrafaktisch die Existenz zentraler handlungsfähiger Akteure auf dem Feld der juristischen Fachdidaktik. Das geht also nicht. Bleibt: Wir müssen auf unsere Adressaten achten. Wenigstens im Kleinen. Reichweitendefizitkompensationsstrategien wären also vielleicht näheren Nachdenkens wert. Gewiss ist die Forderung leichter formuliert als erfüllt: Kann nicht mal eben jemand schnell ein didaktisch modernes Lehrbuch zum Besonderen Verwaltungsrecht schreiben? Solche Wünsche erfüllt eben letztendlich ein Autor oder ein Autorenteam, über deren Arbeitszeit ich nicht aus der Ferne verfügen kann. Aber vielleicht wäre ja schon mit einem einladenden zentralen Forum viel gewonnen. Käme auf einen Versuch an, oder?

Das hier Skizzierte ist nicht nur unautorisiert, sondern auch streng subjektiv, wenn auch möglicherweise anschlussfähig. Weitaus erfreulicher als Beifall wäre indes substanzieller begründeter Widerspruch. 